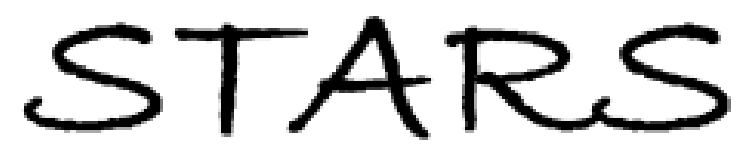

University of Central Florida

STARS

$1-1-2002$

\title{
Existence and properties of quadratic solitons in anisotropic media: Variational approach
}

\author{
Sergey V. Polyakov \\ University of Central Florida \\ George I. Stegeman \\ University of Central Florida
}

Find similar works at: https://stars.library.ucf.edu/facultybib2000 University of Central Florida Libraries http://library.ucf.edu

This Article is brought to you for free and open access by the Faculty Bibliography at STARS. It has been accepted for inclusion in Faculty Bibliography 2000s by an authorized administrator of STARS. For more information, please contactSTARS@ucf.edu.

\section{Recommended Citation}

Polyakov, Sergey V. and Stegeman, George I., "Existence and properties of quadratic solitons in anisotropic media: Variational approach" (2002). Faculty Bibliography 2000s. 3404.

https://stars.library.ucf.edu/facultybib2000/3404

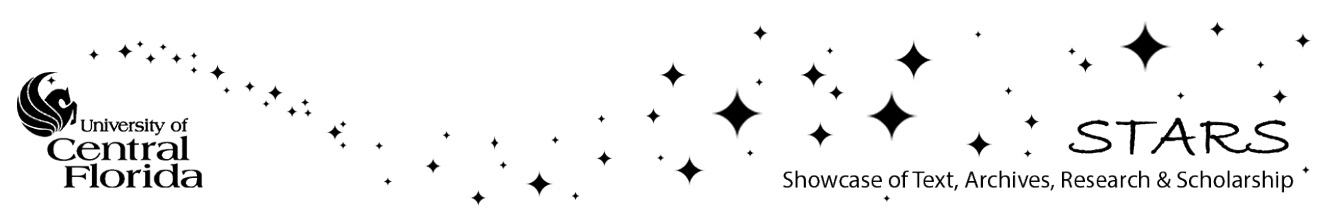




\title{
Existence and properties of quadratic solitons in anisotropic media: Variational approach
}

\author{
Sergey V. Polyakov* and George I. Stegeman \\ School of Optics and CREOL, University of Central Florida, 4000 Central Florida Boulevard, CREOL Building, Orlando, Florida 32816
}

(Received 7 May 2002; published 29 October 2002)

\begin{abstract}
Stationary quadratic solitons associated with second harmonic generation in optically anisotropic media have been investigated both numerically and analytically using the variational approach. The solitons were found to have elliptical shapes, both for the fundamental and second harmonic, and their approximate beam waists and amplitudes as a function of the anisotropy and the soliton parameter were found. The important limits of anisotropic diffraction were compared to the well-known model of isotropic diffraction. The stability of anisotropic solitons was addressed via the Vakhitov-Kolokolov criterion and the regions of parameter space for which the solitons are stable were identified. Direct numerical simulations of the coupled field equations were performed to illustrate the existence, stability, and ellipticity of anisotropic quadratic solitons. In general, good agreement was found between approximate analytical approaches and numerical experiments.
\end{abstract}

DOI: 10.1103/PhysRevE.66.046622

PACS number(s): 42.65.Tg

\section{INTRODUCTION}

Quadratic solitons are supported in media with secondorder (quadratic) nonlinearities due to the strong parametric interaction of two or more waves [1,2]. These effects depend strongly on the dispersion of the material: one needs to approximately phase match at least two optical frequencies in order to observe the strong coupling. In most cases the phase matching requires anisotropic crystal properties. Because different polarizations of the interacting beams would see different dispersion curves, the indices of refraction, e.g., for a fundamental wave $(\mathrm{FW})$ and its second harmonic $(\mathrm{SH})$, can be made equal despite the law of normal dispersion. However, in bulk anisotropic crystals the refractive index surface (and therefore wave vector surface) is no longer spherical but is elliptical in nature. The diffraction coefficient is proportional to the local curvature of the wave vector surface. Therefore, linear diffraction of the interacting optical beams is different for their two transverse dimensions. The difference in the diffraction constants is usually small and there has been no experimental evidence until recently that their anisotropy is important in soliton phenomena. The properties of quadratic solitons, with anisotropic effects neglected, have been explored in detail theoretically, numerically, and experimentally [3-6]. In particular, the variational approach which was successfully used to solve for various properties of optical solitons [7] was applied by Steblina et al. in 1995 to describe stationary quadratic solitons [8].

The formation of multiple solitons observed in recent experiments by Malendevich et al. [9] and Kim and Stegeman [10] has been explained by Polyakov et al. as due to anisotropic diffraction [11]. These experiments dealt with the excitation of the quadratic solitons by a high powered, Gaussian FW. Such excitation conditions are very different from the exact stationary soliton solutions [8]. It turns out that the formation of a stationary soliton with FW input only occurs slowly with distance. In an isotropic medium the circular symmetry is conserved, and therefore a circularly-

*Email address:serge@creol.ucf.edu symmetrical input beam evolves into one soliton with any excess of the input radiation emitted into diffracting rings, regardless of input intensity. However, this is not true for an anisotropic case. In crystals circular symmetry is inherently broken by anisotropy. It was shown recently that the process of beam evolution may lead to the formation of several solitons, aligned on a "preferential" crystal axis even though anisotropic diffraction is small: it is the breaking of the symmetry which matters [11]! The direction of such a preferential axis is determined by the combination of anisotropic diffraction coefficients.

The key questions are now whether the resulting soliton fields that are no longer cylindrically symmetric are stationary (and stable), what is the shape of the field distributions, etc. It is well known, that in the 1D case there is only one set of parameters that leads to an exact analytic solution [1-2]. In the $2 \mathrm{D}$ isotropic diffraction case, there are no analytic solutions, and therefore, variational methods or computer simulations have usually been necessary. The anisotropic 2D case is even more complex, and we do not expect any exact solutions to exist.

\section{VARIATIONAL APPROACH}

Consider an anisotropic medium with a quadratic nonlinearity configured for noncritical phase matching for second harmonic generation with two-dimensional beam cross sections, the case studied experimentally in biaxial crystals [9-11]. The FW and SH index ellipsoids of appropriate polarization just touch along the propagation axis. The necessity for different diffraction coefficients along the two transverse cross-sectional beam directions may be understood from the difference in the local radii of curvature of the wave vector surface along the different crystal axes for both the $\omega$ and $2 \omega$ waves inherent to finite beam propagation along crystal axes in biaxial bulk media (Fig. 1). This can be also derived directly from Maxwell's equations. In crystals, dielectric permittivity is a tensor rather than a scalar. This tensor may be diagonalized by rotation to its principal axes, 


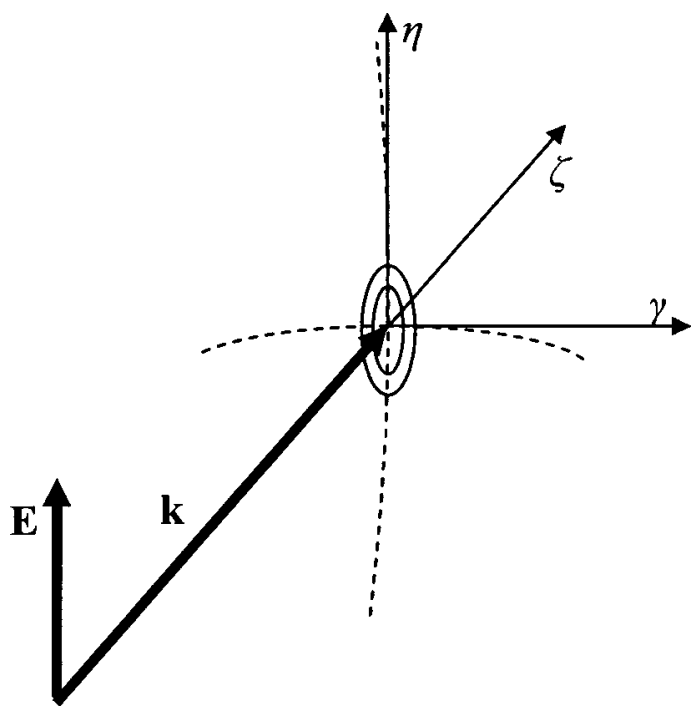

FIG. 1. Linear beam propagation in anisotropic medium. Here $\mathbf{k}$ is the central propagation wave vector of a diffracting beam propagating along the $\zeta$ axis and the dashed lines are the cuts of the wave vector surface in the $\zeta-\eta$ and $\gamma-\zeta$ planes. The ellipses are cuts of the wave vector surface parallel to $\eta$ - $\gamma$ plane and the major and minor diameters are related to the diffraction coefficients along the two crystal axes.

$$
\hat{\boldsymbol{\epsilon}}=\left(\begin{array}{ccc}
\boldsymbol{\epsilon}_{\eta} & 0 & 0 \\
0 & \epsilon_{\gamma} & 0 \\
0 & 0 & \epsilon_{\zeta}
\end{array}\right) .
$$

Let us restrict ourselves to light propagation along one of its principal axes $(\zeta)$ with polarization along an orthogonal axis $(\eta)$ (Fig. 1). Substitution of the light beam in the form $E$ $=A(\eta, \gamma, \zeta) \exp \left(i k_{\zeta} \zeta\right)$ and above permittivity tensor into Maxwell's equations yields the ratio between diffraction coefficients (or constants which multiply the second derivatives of the field $A$ with respect to transverse dimensions) along two perpendicular directions to be equal to $\epsilon_{\eta} / \epsilon_{\zeta}$. From this point on we consider only the case in which all beams are polarized along principal axes. We see that the polarization of light defines the spatial anisotropy in the diffraction coefficient.

Let us now consider the equations for the slowly varying envelopes of the $\omega$ and $2 \omega$ waves.

$$
\begin{gathered}
i \frac{\partial A_{1}}{\partial \zeta}+\left(D_{11} \frac{\partial^{2} A_{1}}{\partial \eta^{2}}+D_{12} \frac{\partial^{2} A_{1}}{\partial \gamma^{2}}\right)=-\Gamma A_{2} A_{1}^{*} \exp (-i \Delta k \zeta), \\
i \frac{\partial A_{2}}{\partial \zeta}+\left(D_{21} \frac{\partial^{2} A_{2}}{\partial \eta^{2}}+D_{22} \frac{\partial^{2} A_{2}}{\partial \gamma^{2}}\right)=-\Gamma A_{1}^{2} \exp (i \Delta k \zeta) .
\end{gathered}
$$

In these equations $D_{11}, D_{12}, D_{21}$, and $D_{22}$ stand for the diffraction of a fundamental wave (FW, first index is 1) and second harmonic ( $\mathrm{SH}$, first index is 2) along the $\eta$ (second index is 1) and $\gamma$ (second index is 2) axes, respectively, $\Gamma$ is proportional to the coefficient of quadratic nonlinearity and $\Delta k$ is the wave vector mismatch between the fundamental $\left(k_{i}, i=1\right)$ and second harmonic $\left(k_{i}, i=2\right)$ wave vectors, i.e. $\Delta k=2 k_{1}-k_{2}$. Depending on the polarization of the input beam, one of the diffraction coefficients for each frequency will be equal to $1 / 2 k_{i}$ and the other one will be different by the ratio $\epsilon_{\eta} / \epsilon_{\zeta}$ (or $\epsilon_{\gamma} / \epsilon_{\zeta}$ ). Note that since these equations have different diffraction coefficients along the beam transverse dimensions $\eta$ and $\gamma$, they do not preserve circular symmetry, as opposed to an isotropic medium in which $D_{11}=D_{12}$ and $D_{21}=D_{22}$.

The problem now is to find the changes in the shape and properties of quadratic solitons due to such anisotropic diffraction. In order to address the existence and approximate shape of the solitons one can employ a variational method, which has been proven to be very effective and valuable in other quadratic soliton problems [8]. First we use standard procedures to normalize the slowly varying envelope equations, which describe the propagation of FWs and $\mathrm{SHs}$ in nonlinear anisotropic media [8],

$$
\begin{gathered}
i \frac{\partial B_{1}}{\partial z}+d_{11} \frac{\partial^{2} B_{1}}{\partial x^{2}}+d_{12} \frac{\partial^{2} B_{1}}{\partial y^{2}}-B_{1}+B_{1}^{*} B_{2}=0 \\
i \sigma \frac{\partial B_{2}}{\partial z}+d_{21} \frac{\partial^{2} B_{2}}{\partial x^{2}}+d_{22} \frac{\partial^{2} B_{2}}{\partial y^{2}}-\alpha B_{2}+\frac{1}{2} B_{1}^{2}=0
\end{gathered}
$$

where the coordinates and other parameters are rescaled with the nonlinear correction to the propagation wave vector for a soliton, $\beta$. Thus $z=\beta \zeta$ is a normalized propagation coordinate, $x=\left(2 \beta k_{1}\right)^{1 / 2} \eta$ and $y=\left(2 \beta k_{1}\right)^{1 / 2} \gamma$ are normalized transverse coordinates, and the $B_{i}$ are normalized $\mathrm{FW}(i$ $=1)$ and $\mathrm{SH}(i=2)$ fields. $\sigma=k_{2} / k_{1}$ is close to 2 for nearphase-match operation and the normalized anisotropic diffraction terms $d_{i j}=2 k_{i} D_{i j}$ are close to unity (and greater than zero). The subscript " $j$ " refers to the transverse directions $x$ or $y$. The original electric fields are related to the normalized ones by $A_{1}=(\beta / \Gamma \sqrt{2 \sigma}) B_{1} \exp (i \beta \zeta)$ and $A_{2}$ $=(\beta / \Gamma) B_{2} \exp [i(2 \beta+\Delta k) \zeta]$. Finally, $\alpha=(2 \beta+\Delta k) \sigma / \beta$ is the soliton parameter describing the ratio between the wave vector mismatch $\Delta k$ and $\beta$. The requirement of exponential localization of the solitons necessitates $\alpha>0$.

Unlike Kerr-like solitons, this problem is inherently two dimensional and cannot be generally reduced to quasicircular symmetry by further renormalization of the normalized transverse coordinates $x$ and $y$, i.e., there is no cylindrical symmetry because the $d_{i j}$ are not equal to each other. These equations are formally similar to ones describing quadratic light bullets $[12,13]$, used to discuss the properties of onedimensional, spatio-temporal quadratic solitons with "anisotropy" necessitated by dispersion. Our case involves physical limitations on the size of the anisotropy found in nature that allows the introduction of further analytical approximations. Also, if the transverse axes $x$ and $y$ are rescaled, the soliton parameter $\alpha$ as well as the field normalization will take different forms from the most common ones [8].

For stationary solutions, i.e., no dependence of the amplitudes on propagation coordinate $z$ 


$$
\begin{aligned}
& d_{11} \frac{\partial^{2} B_{1}}{\partial x^{2}}+d_{12} \frac{\partial^{2} B_{1}}{\partial y^{2}}-B_{1}+B_{1}^{*} B_{2}=0 \\
& d_{21} \frac{\partial^{2} B_{2}}{\partial x^{2}}+d_{22} \frac{\partial^{2} B_{2}}{\partial y^{2}}-\alpha B_{2}+\frac{1}{2} B_{1}^{2}=0 .
\end{aligned}
$$

Following the procedure outlined by Steblina et al. [8], we introduce the Lagrangian, given by

$$
\begin{aligned}
L= & \frac{1}{2} \int_{-\infty}^{+\infty} \int_{-\infty}^{+\infty} d x d y\left\{\left(d_{11}\left(\frac{\partial B_{1}}{\partial x}\right)^{2}+d_{12}\left(\frac{\partial B_{1}}{\partial y}\right)^{2}\right.\right. \\
& \left.\left.+d_{21}\left(\frac{\partial B_{2}}{\partial x}\right)^{2}+d_{22}\left(\frac{\partial B_{2}}{\partial y}\right)^{2}\right)+B_{1}^{2}+\alpha B_{2}^{2}-B_{1}^{2} B_{2}\right\},
\end{aligned}
$$

and approximately solve the resulting Euler-Lagrangian equations. Allowing the soliton to have an elliptical rather than a circular form, we introduce trial functions of the form,

$$
B_{1}=\Psi_{1} \mathrm{e}^{-b_{11} x^{2}-b_{12} y^{2}} ; \quad B_{2}=\Psi_{2} \mathrm{e}^{-b_{21} x^{2}-b_{22} y^{2}} .
$$

These functions are used to turn the variational problem $\delta L$ $=0$ into a set of algebraic equations obtained by taking all first derivatives of $L$ with respect to the parameters $\Psi_{i}$ and $b_{i j},(i ; j)=1,2$. Substituting the trial function into the Lagrangian yields a system of six equations. After simplification the amplitudes of the elliptical Gaussian solutions assumed are found to be

$$
\begin{gathered}
\Psi_{2}^{2}=\frac{\left(b_{11} d_{11}+b_{12} d_{12}+1\right)}{2 \sqrt{b_{11} b_{12}}} \sqrt{\left(2 b_{11}+b_{21}\right)\left(2 b_{12}+b_{22}\right)}, \\
\Psi_{1}=\left\{\Psi_{2} \frac{\left(b_{21} d_{21}+b_{22} d_{22}+\alpha\right)}{\sqrt{b_{21} b_{22}}}\right. \\
\left.\times \sqrt{\left(2 b_{11}+b_{21}\right)\left(2 b_{12}+b_{22}\right)}\right\}^{1 / 2} .
\end{gathered}
$$

The soliton waists are given by

$$
\begin{gathered}
b_{21}=\frac{4 b_{11}^{2} d_{11}}{1-b_{11} d_{11}+b_{12} d_{12}}, \\
b_{22}=\frac{4 b_{12}^{2} d_{12}}{1+b_{11} d_{11}-b_{12} d_{12}}, \\
b_{11}=\frac{b_{21}}{2}\left(1+\frac{4 b_{21} d_{21}}{\alpha-b_{21} d_{21}+b_{22} d_{22}}\right), \\
b_{12}=\frac{b_{22}}{2}\left(1+\frac{4 b_{22} d_{22}}{\alpha+b_{21} d_{21}-b_{22} d_{22}}\right) .
\end{gathered}
$$

The beam waists must be positive so that $\left|b_{11} d_{11}-b_{12} d_{12}\right|$ $<1$ and $\left|b_{21} d_{21}-b_{22} d_{22}\right|<\alpha$. It is possible to express the $\mathrm{SH}$ beam waists in terms of the FW beam waists and independent material parameters, and vice versa. However, further elimination of variables leads to very complex equations of fifth order.

It is instructive to examine several special cases before proceeding with the most general treatment. For isotropic diffraction, i.e., setting all diffraction parameters $d_{i j}$ to 1 , the set of completely independent equations first found in Ref. [8] is obtained, namely,

$$
b_{11}=b_{12}, \quad b_{21}=b_{22}, \quad-\alpha+2 \alpha b_{11}+32 b_{11}^{3}=0 .
$$

Here one needs to retain only positive real roots due to physical restrictions on beam waist. Another interesting solution arises for $b_{11} d_{11}-b_{12} d_{12}=0$, which means that the solitons are elliptical, and their ellipticity is simply proportional to the anisotropy of the FW diffraction coefficients. This condition requires $d_{11} d_{22}=d_{12} d_{21}$, which basically states that for this particular case there exists a transformation (i.e., rescaling of transverse coordinates $x$ and $y$ ) which transforms the set of two bounded Eqs. (1) to circular symmetry. In this case the variables in the equations for beam sizes $b_{i j}$ are easily separable which leads to an equation similar to Eq. (2), namely,

$$
-\alpha+2 \alpha d_{11} b_{11}+32 d_{11}^{2} d_{21} b_{11}^{3}=0 .
$$

This is an important result which shows that the anisotropic soliton parameters are expected to depend on the phase mismatch, similar to the dependence found for isotropic soliton parameters. Also, if one is to employ numerical methods to solve the full set of algebraic equations, the suggested initial guess would be given by Eq. (2) or Eq. (3). It is worth noting to observe that for any $\alpha>0, d_{11}>0$ and $d_{21}>0$, a $b_{11}>0$ does exist. Subsequently this special case will be referred to as quasi-isotropic.

The most general case is now considered. Treating the parameter $\alpha$ as a variable, introducing the substitutions $b_{12} d_{12}=l$ and $b_{11} d_{11}=n$, and choosing $n$ to be a second variable and $l$ to be a parameter, the "variables" can be separated and the set of solutions found. For the material parameters $d_{11}$ and $d_{12}$, solutions of different ellipticity are sought by varying the parameter $l$. This leads to

$$
\alpha=\frac{2 n^{2}}{n-l}\left\{\frac{d_{21}}{d_{11}}\left(1+\frac{2(n+1)}{(n-l-1)}\right)-\frac{d_{22}}{d_{12}}\left(3-\frac{2(n+2)}{(n-l+1)}\right)\right\}
$$

and

$$
\begin{aligned}
0= & \frac{(-l-n-1)}{n-l}\left[\frac{d_{22}}{d_{12}}(n-l-1)(3 n-l-1) l^{2}\right. \\
& \left.-\frac{d_{21}}{d_{11}}(n-l+1) n^{2}(n-3 l+1)\right] .
\end{aligned}
$$




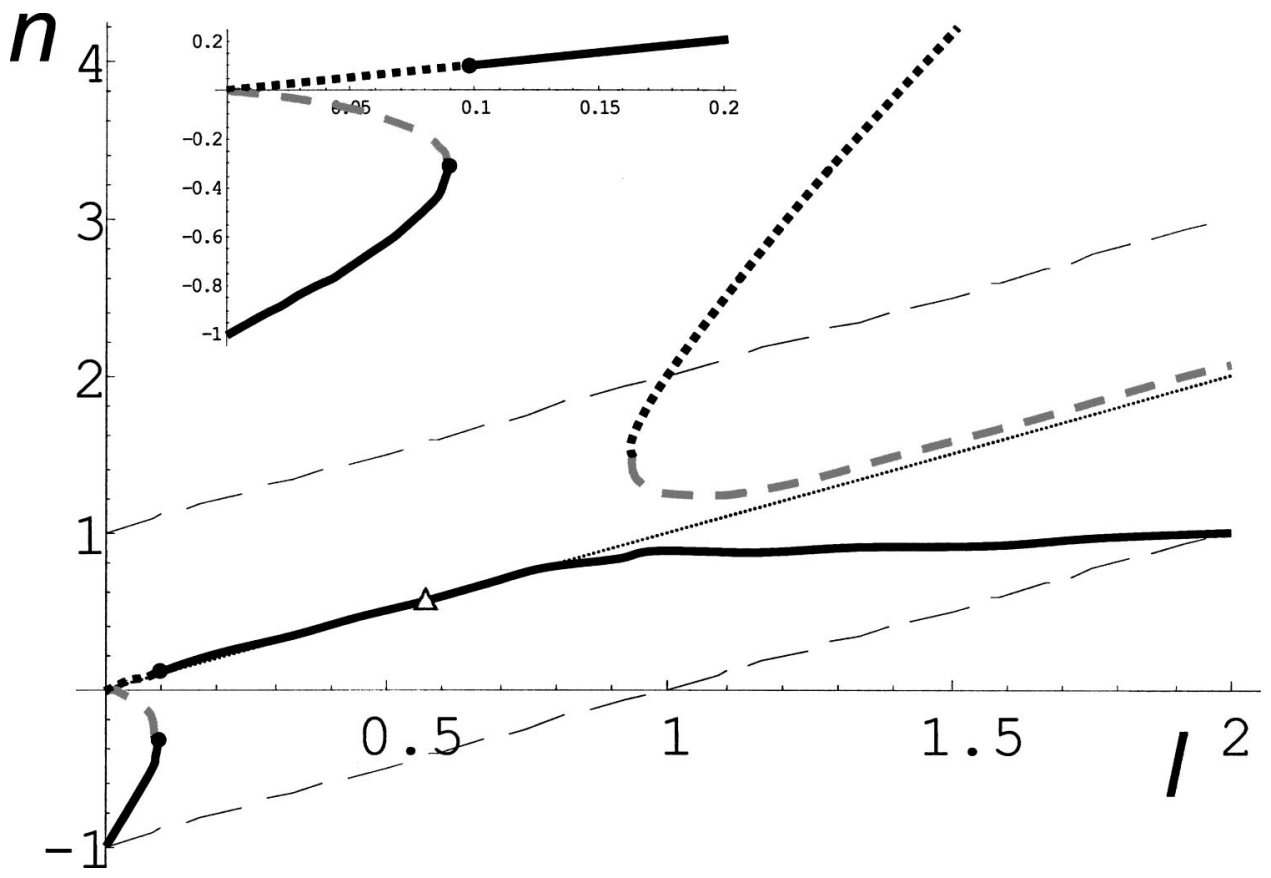

FIG. 2. Variation in $n$ as a function of $l$ for $d_{21} / d_{11}=1$ and $d_{22} / d_{12}=1.1$. Thin dashed lines, physical limits for $n$ and $l$. Thin dotted line, quasi-isotropic dependence. Thick dashed line, unphysical branch. Thick dotted line, branch containing unphysical and physical (but ultimately unstable) solutions. The region near the origin is expanded in the inset. Physical solutions are located from the origin up to the black dot. The thick solid line, which is discontinuous between the two black dots, contains both unphysical and physical (partly stable and partly unstable) solutions. Its physical solutions lie on the line between the black dot and the white triangle. The soliton parameter $\alpha$ varies monotonically from 0 at the origin to infinity at the triangle position.

The last equation yields five different solutions for any given l. However, most of the solutions are unphysical. Indeed, both $n$ and $l$ must be real positive values, $|n-l|<1$, and the parameter $\alpha$ also is expected to be positive to insure selftrapping, by analogy to the isotropic case. Note that the set of solutions for $n-l=0$ requires special treatment and coincides with the "quasi-isotropic" case treated above. Furthermore, two of the solutions do not match the criteria for any values of parameters and are considered unphysical.

The typical variation of $n$ as a function of $l$ for $d_{21} / d_{11}$ $=1$ and $d_{22} / d_{12}=1.1$ in the real plane is shown in Fig. 2, along with the largest difference between $n$ and $l$ (thin dashed lines) acceptable by the physical constraints that both $n$ and $l$ must be real positive values and $|n-l|<1$. The thin dotted line is for $n=l$. The exclusion of nonphysical solutions leaves only one continuous set of physical solutions, made up of partial combinations of two different branches. These physical solutions start at the origin as a dotted line and continue smoothly (at the solid dot) as a solid line up to $l \approx 0.55$ (marked by a triangle). Each pair of physical parameters, $n$ and $l$, determines a unique $\alpha$, which varies from 0 to positive infinity as $l$ varies from 0 to $\approx 0.55$. The continuation of this set for $l>\approx 0.55$ is unphysical, because $\alpha$ becomes negative. Note that the set of physical solutions does not deviate much from the "quasi-isotropic" solution for all physically reasonable ratios (from about 0.7 to about 1.4 ) of $d_{21} / d_{11}$ and $d_{22} / d_{12}$. This suggests another useful substitution, $m=n-l$ and an approximation to the equations that retains only linear terms with respect to $m$. For Eq. (5) this yields the simple first-order equation relating $l$ to $n$ (after substituting back $m=n-l$ ),

$$
l=\frac{2\left[\frac{d_{21}}{d_{11}}+\frac{d_{22}}{d_{12}}\right] n^{3}-2 \frac{d_{21}}{d_{11}} n^{2}-\left[\frac{d_{21}}{d_{11}}+\frac{d_{22}}{d_{12}}\right] n}{2\left[\frac{d_{21}}{d_{11}}+\frac{d_{22}}{d_{12}}\right] n^{2}+2\left[\frac{d_{22}}{d_{12}}-2 \frac{d_{21}}{d_{11}}\right] n-2 \frac{d_{22}}{d_{12}}} .
$$

After applying the same approximation to Eq. (4), the soliton's waist as a function of the parameters $\alpha$ and $d_{i j}$ is given by

$$
-\alpha+2 \alpha n+4\left(\frac{d_{21}}{d_{11}}-\frac{d_{22}}{d_{12}}\right) n^{2}+16\left(\frac{d_{21}}{d_{11}}+\frac{d_{22}}{d_{12}}\right) n^{3}=0 .
$$

Formally, Eq. (7) includes both Eqs. (2) and (3), even though it is derived under the condition that $n-l \neq 0$ (or $d_{11} d_{22}$ $\left.\neq d_{12} d_{21}\right)$. As discussed before, there is only one real positive solution for each $\alpha>0$ and $d_{i j}>0$.

\section{STABILITY}

The stability issue was addressed by employing the Vakhitov-Kolokolov criterion for soliton stability [14]. The dependence of the Hamiltonian

$$
\begin{aligned}
H= & \iint\left\{d_{11}\left|\frac{\partial B_{1}}{\partial x}\right|^{2}+d_{12}\left|\frac{\partial B_{1}}{\partial y}\right|^{2}+d_{21}\left|\frac{\partial B_{2}}{\partial x}\right|^{2}+d_{22}\left|\frac{\partial B_{2}}{\partial y}\right|^{2}\right. \\
& \left.-\frac{1}{2}\left(B_{1}^{* 2} B_{2}+B_{1}^{2} B_{2}^{*}\right)\right\} d \tilde{x} d \tilde{y}
\end{aligned}
$$

on the total energy 

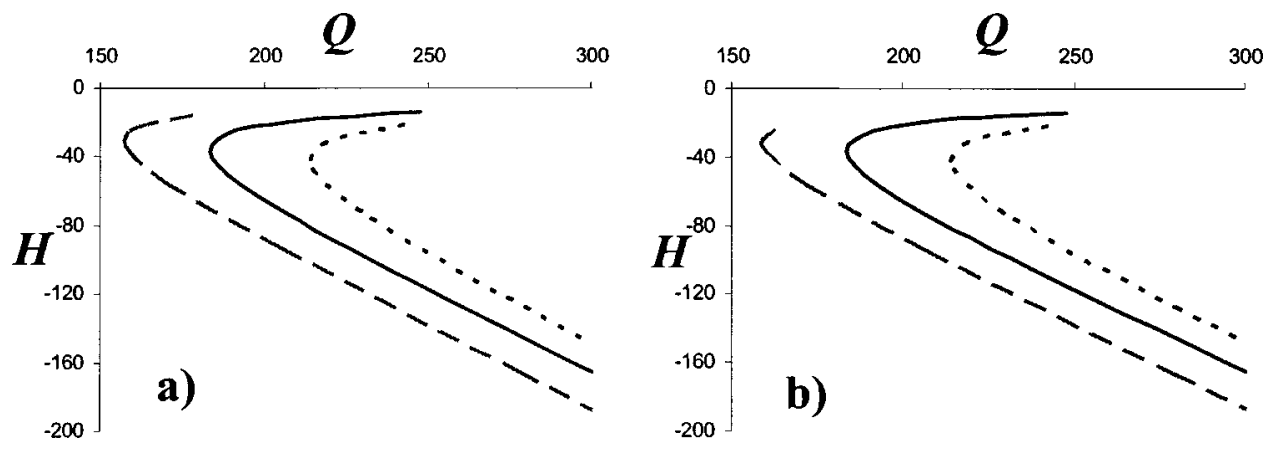

FIG. 3. Hamiltonian versus energy for anisotropic solitons with the isotropic limit given by the solid line. (a) Examples of the quasi-isotropic limit, $d_{11}=d_{21}$ $=1.3, d_{21}=d_{22}=1$ (dashed); $d_{11}$ $=d_{21}=0.7, d_{21}=d_{22}=1$ (dotted); (b) Examples of substantially anisotropic cases, $d_{11}=1.3, d_{12}=1$, $d_{21}=0.7, \quad d_{22}=1 \quad$ (dashed); $d_{11}$ $=0.7, d_{21}=1, d_{21}=1.3, d_{22}=1$ (dotted).

$$
Q=\iint\left(\left|B_{1}\right|^{2}+2 \sigma\left|B_{2}\right|^{2}\right) d \tilde{x} d \tilde{y}
$$

for different amounts of anisotropic diffraction was compared with the isotropic dependence. It is obvious that two physically different cases need to be considered individually. The curves shown in Fig. 3(a) are for the cases $d_{11}>d_{12}$ together with $d_{21}>d_{22}$, or $d_{11}<d_{12}$ together with $d_{21}$ $<d_{22}$, which is typical for an ooo geometry (quasi-phase matched solitons). Solitons of this kind can be approximated in many cases by the quasi-isotropic solitons discussed above. Indeed, for periodically poled KTP, the diffraction anisotropy is about $13 \%$ for the $\mathrm{FW}$ and $11 \%$ for the $\mathrm{SH}$. That is, $\epsilon_{\eta} / \epsilon_{\zeta}$ will be equal to 1.106 for $\mathrm{FF}$ and 1.125 for $\mathrm{SH}$ [15]. The multiple soliton generation mechanism in such systems is currently being studied and is beyond the scope of this paper. Fig. 3(b) presents the $H(Q)$ dependence for orthogonal orientations of the long axes of the diffraction ellipse for the FW and the SH. This is usually the case for ooe SHG geometries (Type I solitons). The beam propagation in such the materials was discussed in Ref. [11], and exhibits novel behavior when compared to isotropic diffraction. Usually the soliton is excited via a single Gaussian-shaped FW beam, which is substantially different from the stationary soliton solution, and the formation of high energy solitons is affected by beam deformation due to anisotropic diffraction and results in multiple soliton generation. It turns out, however, that both cases yield comparable behavior in an $H(Q)$ plot. This result shows that despite fundamental differences in multiple soliton generation mechanisms the solitons in both cases are similar. In all plots, the $\alpha$ parameter grows along the curve starting from its right-uppermost corner. Thus the necessary stability criterion $d H / d Q<0$ is satisfied for sufficiently large $\alpha$ and the shape, and main features of the $H(Q)$ curves are very similar to the isotropic case. It is very important to note that the branch splitting found with the variational approach and plotted in Fig. 2 does not constitute a change of slope for the $H(Q)$ curve, yielding the same $H(Q)$ dependence as in the isotropic case [8]. Therefore, the stability puts another constraint on the range of acceptable soliton widths. Specifically for physically acceptable anisotropies, the physical solutions from the origin to at least the solid circle in Fig. 2 are unstable with the stability range determined by the details of the anisotropy.

\section{NUMERICAL SIMULATIONS}

In order to support our analytical findings, we performed a series of direct numerical simulations of the normalized coupled equations for a large (relative to that found in most phase-matchable crystals) diffraction deformation of about $30 \%$ to emphasize the noncircular nature of the soliton fields. We use a standard split-step Fourier method to solve (1) for an initial field distribution based on the approximate soliton solutions. As an example, for the soliton parameter $\alpha=10$ we compare the propagation of both quasi-isotropic and anisotropic solitons defined by $d_{11}=d_{21}=0.7, d_{12}=d_{22}=1$, and $d_{11}=d_{22}=0.7, d_{12}=d_{21}=1$, respectively. According to the predictions of the variational method combined with the Vakhitov-Kolokolov criterion we should have stable soliton propagation in both cases.

First, we discuss propagation of a quasi-isotropic soliton with $\alpha=10$. The propagation of the soliton found via the variational method is not stationary at the input because of this technique's inherent approximations [Fig. 4(a)], but evolves towards a stationary soliton. We see that the output fields are slightly elliptical. The ratio of the half-widths at half maximum (HWHM) along the axes for the FW is the same as for the $\mathrm{SH}$, around 0.87 [Fig. 4(b)]. This value persists with small changes in beam size due to nonstationary propagation. This result can be obtained from a renormalization of transverse dimensions which renders the coefficients for the diffraction terms equal to unity in Eq. (1), and also from the variational method, both of which give 0.84 for the beam waist ratio (calculated from $\sqrt{b_{12} / b_{11}}$ ). Thus we see that the 2D numerical method is consistent (to within 4\%) with the approximate analytics and preserves the quasicircular symmetry of the problem.

The propagation results for anisotropic solitons with $\alpha$ $=10$, again based on the initial solutions used for the variational technique, are also near the input not strictly stationary [Fig. 5(a)], similar to the quasi-isotropic case. The approximate ratio of the beam waists along the two axes is 0.87 for the FW and 0.96 for the SH [Fig. 5(b)]. The variational approach gives 0.86 and 0.92 correspondingly. Because the intensity of the FW component is substantially higher than the $\mathrm{SH}$ and the waves are strongly coupled, the deviation from a circular beam shape is in the same direction in space for both components, and not in orthogonal directions as expected from linear optics (decoupled waves) for $d_{12} / d_{11}>1$ and $d_{22} / d_{21}<1$. Therefore, we note that a variational approxima- 
a)
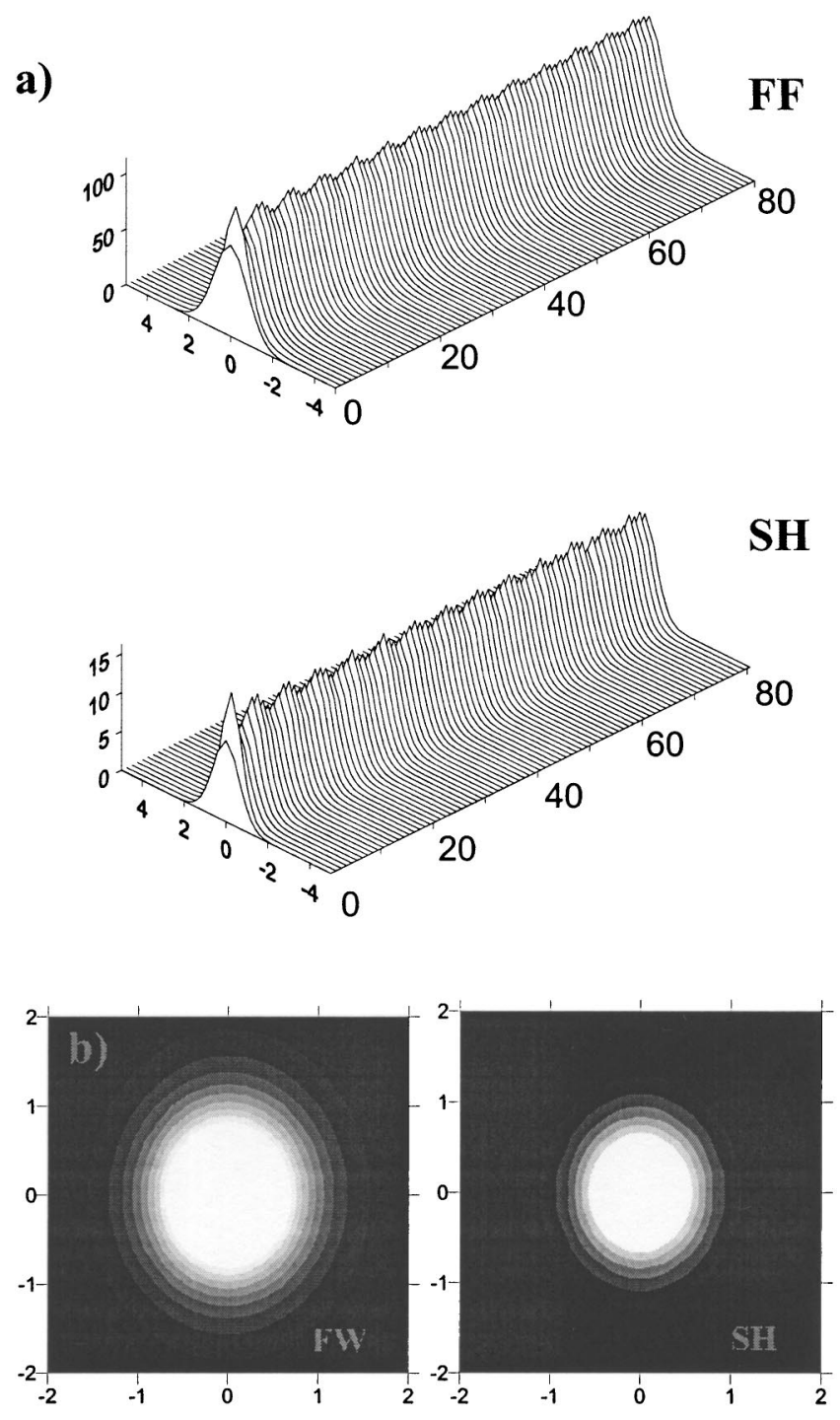

FIG. 4. Soliton propagation and shapes after propagation in a nonlinear quasi-isotropic medium with $\alpha=10, d_{11}=d_{21}=0.7$, and $d_{12}=d_{22}=1$. (a) intensity distribution along $y$ axis during propagation; (b) output profile and evolution of the FW and $\mathrm{SH}$.

tion describes the relative orientation of the long semiaxes of the elliptical soliton, although it fails to predict the exact beam waist at HWHM. The latter can be explained by the fact that the trial functions (Gaussians) used in the variational method differ from the exact soliton shape. This shows that the variational method developed here predicts correctly the existence and properties of anisotropic solitons. Note that $|m|$ is less than 0.05 in this case, which makes the propagation of anisotropic solitons given by the approximate formulas (6) and (7) valid.

The small diffraction anisotropy available for physically realizable crystals leads to only weak ellipticity in the field profiles and it is not surprising that it has not been reported a)
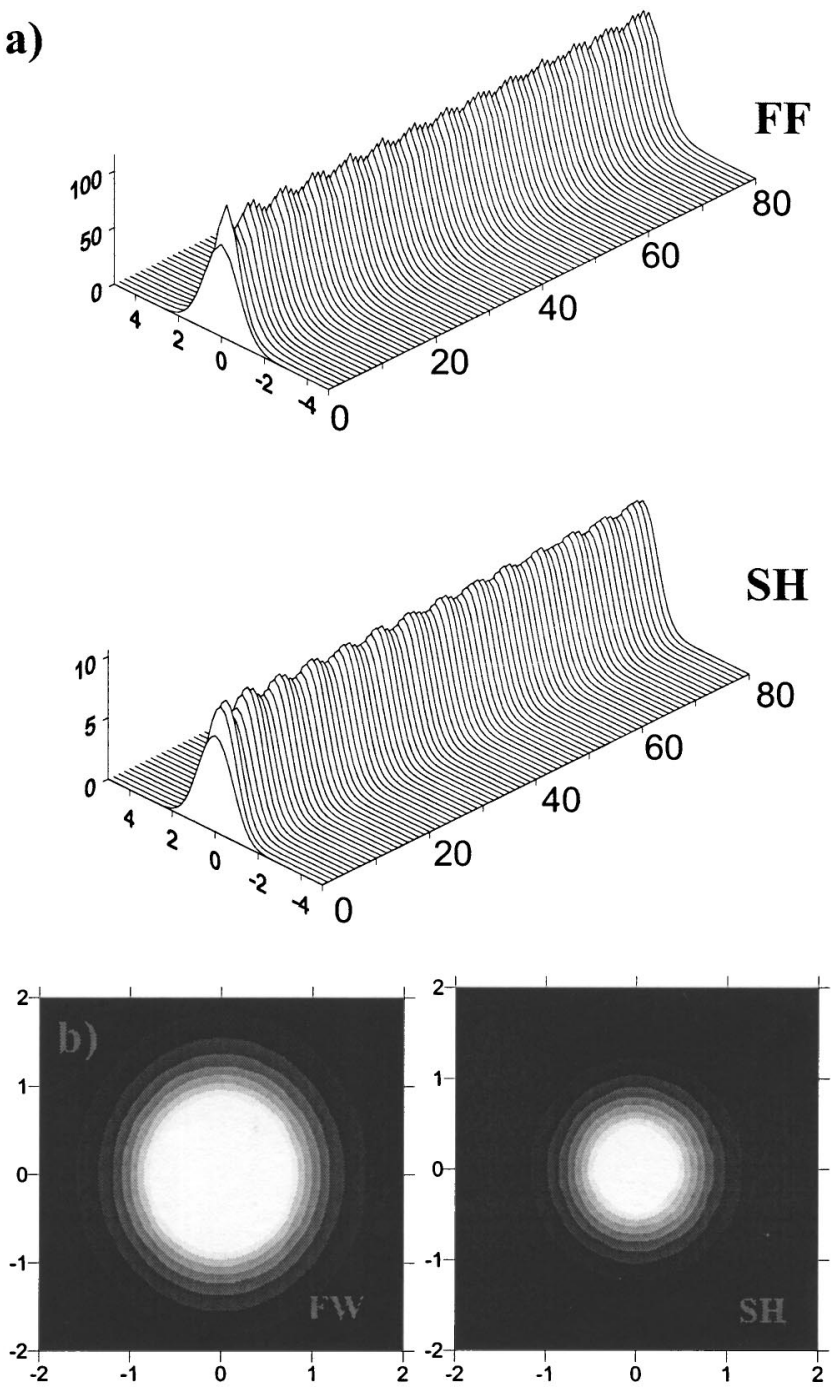

FIG. 5. Soliton propagation and shapes after propagation in a nonlinear essentially anisotropic medium with $\alpha=10, d_{11}=d_{22}$ $=0.7$, and $d_{12}=d_{21}=1$. (b) intensity distribution along $y$ axis during propagation; (b) output profile and evolution of the FW and SH.

experimentally. However, the effect of diffraction anisotropy does manifest itself clearly in multisoliton generation in a number of noncritically phase-matched second harmonic geometries.

\section{CONCLUSIONS}

We showed, both analytically and numerically, that quadratic solitons in optically anisotropic media exist, are stable, and have an elliptical shape. We also explored the multiple solutions obtained by a variational approach to find the uniqueness of the soliton solution for any given set of $d_{i j}$ (diffraction coefficients) and $\alpha$ (soliton parameter depending on intensity and wave vector mismatch). Depending on $\alpha$, these solutions may be stable or unstable. 
[1] Y. Karamzin and A. Sukhorukov, Zh. Eksp. Teor. Fiz Pis'ma Red. 20, 734 (1974) [JETP Lett. 20, 339 (1974)].

[2] Y. Karamzin and A. Sukhorukov, Zh. Eksp. Teor. Fiz. 68, 834 (1975) [Sov. Phys. JETP 41, 414 (1976)].

[3] W. Torruellas, Z. Wang, D. Hagan, E. Van Stryland, G. Stegeman, L. Torner, and C. Menyuk, Phys. Rev. Lett. 74, 5036 (1995).

[4] R. Schiek, Y. Baek, and G. I. Stegeman, Phys. Rev. E 53, 1138 (1996)

[5] V. Buryak and Yu. S. Kivshar, Opt. Lett. 13, 1612 (1994).

[6] L. Torner, Opt. Commun. 114, 136 (1995).

[7] D. Anderson, Phys. Rev. A 27, 3135 (1983).

[8] V. V. Steblina, Y. S. Kivshar, M. Lisak, and B. A. Malomed, Opt. Commun. 118, 345 (1995).
[9] R. Malendevich, L. Jankovic, S. Polyakov, R. Fuerst, G. I. Stegeman, Chr. Bosshard, and P. Gunter, Opt. Lett. 27, 631 (2002).

[10] H. Kim and G. Stegeman (private communication).

[11] S. Polyakov, R. Malendevich, L. Jankovic, G. Stegeman, Chr. Bosshard, and P. Gunter, Opt. Lett. 27, 1049 (2002).

[12] B. A. Malomed et al., Phys. Rev. E 56, 4725 (1997).

[13] D. Mihalache et al. Opt. Commun. 152, 365 (1998).

[14] N. G. Vakhitov and A. A. Kolokolov, Radiophys. Quantum Electron. 16, 783 (1973).

[15] V. G. Dmitriev, G. G. Gurzadyan, and D. N. Nikogosyan, Handbook of Nonlinear-Optical Crystals (Springer-Verlag, Berlin, 1999), p. 113, for the flux-grown KTP. 